\title{
REUTILIZAÇÃO DE SERINGAS DESCARTÁVEIS: FREQÜÊNCIA E CUSTOS PARA ADMINISTRAÇÃO DE INSULINA NO DOMICÍLIO
}

\author{
Carla Regina de Souza Teixeira ${ }^{1}$ \\ Maria Lúcia Zanetti ${ }^{2}$ \\ Kátia Prado Ribeiro ${ }^{3}$
}

Teixeira CRS, Zanetti ML, Ribeiro KP. Reutilização de seringas descartáveis: freqüência e custos para administração de insulina no domicílio. Rev. Latino-am. Enfermagem 2001 setembro-outubro; 9(5):47-54.

Estudo tipo "survey" com o objetivo de analisar a freqüência e os custos de reutilização de seringas descartáveis para administração de insulina no domicílio. Foram entrevistados 113 portadores de diabetes atendidos em um hospital público de grande porte, no interior do estado de São Paulo - SP. Os resultados permitiram concluir que: na população estudada a freqüência de reutilização das agulhas variou de uma a 20 vezes, e das seringas de uma a 60 vezes, sendo que as maiores freqüências foram de 4 seguido de 3 vezes o uso da mesma seringa e agulha. Com a prática de reutilização de seringas de insulina por 4 vezes, conforme referido pela maioria dos sujeitos neste estudo, haveria uma redução de custos na aquisição de seringas na ordem $74,68 \%$. Esses resultados apontam para a necessidade urgente de estudos sistematizados acerca dos riscos e benefícios destas práticas, oferecendo subsídios para a revisão da portaria da DIMED 04/86.

PALAVRAS CHAVE: diabetes mellitus, insulinoterapia, seringas, equipamentos descartáveis

\section{RE-UTILIZATION OF DISPOSABLE INSULIN SYRINGES: FREQUENCY AND BENEFITS OF INSULIN ADMINISTRATION AT HOME}

The present survey has the purpose to analyze the frequency and costs of the re-utilization of disposable syringes for insulin administration at home. One hundred and thirteen patients with diabetes assisted by a public hospital in a city in the interior São Paulo State, Brazil, were interviewed. The findings showed that regarding the studied population, the frequency of needles re-utilization varied from 1 to 20 times, and syringes re-utilization from 1 to 60 times. The greatest frequencies in the use of the same syringe or needle were of four times followed by three times. Re-utilizing the same syringe four times resulted in a cost reduction of $74.68 \%$. The results pointed out the need for a legislation review as well as for new studies on the risks and benefits arising from this practice.

KEY WORDS: diabetes mellitus, insulin therapy, syringes, disposable equipment

\section{REUTILIZACIÓN DE JERINGAS DESECHABLES: FRECUENCIA Y COSTOS PARA LA ADMINISTRACIÓN DE INSULINA EN EL HOGAR}

Estudio tipo "survey" con el objetivo de analizar los frecuencia y los costos de reutilización de jeringas desechables para la administración de insulina en el hogar. Fueron entrevistados 113 portadores de diabetes atendidos en un hospital público de gran porte, en el interior del Estado de São Paulo-SP. Los resultados permitieron concluir que: en la población estudiada la frecuencia de reutilización de las agujas varió de 1 a 20 veces, y de las jeringas de 1 a 60 veces, siendo que las mayores frecuencias fueron de 4 veces, seguidas de 3 veces en el uso de la misma jeringa y aguja. Con la práctica de reutilización de jeringas de insulina por 4 veces, conforme es referido por la mayoría de los sujetos en este estudio, habría una reducción de costos en la adquisición de jeringas en un orden del $74,68 \%$. Estos resultados muestran la necesidad urgente de estudios sistematizados a cerca de los riesgos y beneficios de éstas prácticas, así como también, ofrece un importante aporte para la revisión de la circular de la DIMED 04/86.

PALABRAS CLAVES: diabetes mellitus, insulino-terapia, jeringas, equipos desechables

\footnotetext{
${ }^{1}$ Enfermeira, doutoranda da Escola de Enfermagem de Ribeirão Preto da Universidade de São Paulo, vinculada ao Programa de Enfermagem Fundamental;

${ }^{2}$ Professor Doutor da Escola de Enfermagem de Ribeirão Preto da Universidade de São Paulo, Centro Colaborador da OMS para o desenvolvimento da pesquisa em enfermagem, e-mail: zanetti@eerp.usp.br; ${ }^{3}$ Enfermeira, bolsista de Apoio Técnico do CNPq
} 


\section{INTRODUÇÃO}

$\boldsymbol{U}_{\mathrm{m}}$ dos aspectos que despertado nossa atenção na assistência à pessoa diabética é a insulinoterapia, em especial, o instrumental utilizado na administração de insulina, ou seja, a reutilização das seringas descartáveis.

Sabemos que existe a recomendação de uso único na embalagem original da seringa, considerando que as condições de fabricação e esterilidade são garantidas pelos fabricantes apenas no primeiro uso. Esta recomendação foi exigida pela Vigilância Sanitária de Medicamentos (DIMED) em sua portaria $\mathrm{n}^{0} 3$ de 07 de fevereiro de $1986^{(1)}$.

As condições de esterilidade de um artigo médico hospitalar são garantidas pelos fabricantes no primeiro uso; a reutilização da seringa descartável na aplicação de insulina pode transmitir agentes infecciosos aos portadores de diabetes ${ }^{(2)}$.

No entanto, a literatura internacional e nacional ${ }^{(3-6)}$ mostram índices zero ou baixos de contaminação em culturas de seringas descartáveis reutilizadas.

O que nos preocupa é que os estudos analisados enfocam diferentes metodologias e variáveis gerando dificuldades nas comparações. Temos também poucos estudos internacionais ${ }^{(7-8)}$ que mostram casos de abcessos e complicações locais relacionados com a reutilização de seringas.

\section{REVISÃO DA LITERATURA}

$\mathrm{Na}$ literatura nacional e internacional verificamos que a reutilização de seringas descartáveis tem implicações para o uso seguro e satisfatório para qual o produto foi fabricado.

Quanto às alterações físicas e químicas do material, os portadores de diabetes estão sujeitos ao risco de infecção, pois as agulhas depois de serem reutilizadas por um certo período mostramse danificadas, rombudas e acumulam detritos em seu lumen ${ }^{(9)}$.

Quanto ao procedimento de reutilização da seringa de insulina, esta deveria ser recolocada na embalagem original após a sua utilização, e armazenada na geladeira junto com o frasco de insulina ${ }^{(10)}$. Em contrapartida, armazenar as seringas na geladeira, evita o crescimento do Mycobacteria, mas não assegura a eliminação de outros microrganismos existentes ${ }^{(11)}$.

Em relação à transmissão de agentes infecciosos no material utilizado para aplicação de insulina, foram encontrados culturas de Bacillus sp e Clostridium fallax ${ }^{(12)}$. Ao analisar culturas de seringas reutilizadas outros autores ${ }^{(10)}$ encontraram Staphylococcus albus. Vale destacar que a principal proteção de abscessos nos locais de aplicação de insulina são os aditivos contidos na insulina. Estes aditivos são efetivos na prevenção do crescimento de Staphylococcus aureus $^{(11)}$

Neste sentido, procurando identificar o crescimento bacteriano em frascos de insulinas, um amplo estudo bacteriostático ${ }^{(3)}$ demonstrou que não houve crescimento bacteriano e nem evidência de infecção ou irritação na pele dos locais de aplicação de insulina. Num outro estudo prospectivo ${ }^{(13)}$ também não foi observada infecção nos locais de aplicação da injeção.

No que refere as complicações locais ${ }^{(7)}$ há um relato de caso de portador de diabetes tipo 1 que apresentou múltiplos abcessos no local de aplicação de insulina causado por Mycobacterium chelonei, um raro patógeno humano. Um estudo retrospectivo analítico constatou que não houve alterações significativas entre os que reutilizavam a seringa descartável comparada à de vidro ${ }^{(14)}$.

Em cultura de restos de insulina de pacientes que reutilizavam as seringas descartáveis não foram observados crescimento de microrganismos e não foram observadas complicações locais ou sistêmicas ${ }^{(6)}$. Conclui-se que as seringas descartáveis de insulina poderiam ser reutilizadas até sete vezes, desde que fosse preservado a conservação adequada da seringa até a próxima aplicação, sendo um método útil para diminuir os custos com o tratamento do diabetes tipo $1 \mathrm{em}$ uma população de baixo poder aquisitivo. Outros autores recomendam ${ }^{(15)}$ a reutilização por até seis vezes, considerando que acima desta freqüência, as agulhas tornaram-se rombudas e as aplicações de insulina dolorosas.

Um estudo ${ }^{(16)}$ realizado sobre a preferência por utilizar seringas de vidro ou descartáveis na aplicação de insulina constatou que "se os fabricantes e os profissionais de saúde estavam assegurando que as seringas e agulhas poderiam ser reutilizadas com segurança por duas ou três semanas, eles deveriam então estar preparados para recomendar esta prática junto ao governo."

A distribuição gratuita de seringas baseada no único uso poderia representar um gasto super-estimado e que talvez 0 excedente de verbas poderia ser aplicado em fitas para mensuração da glicemia, considerando a prática de que os portadores de diabetes reutilizam a mesma seringa e agulha por longos períodos ${ }^{(4,17)}$.

Coloca-se em questão que, se de fato não há perigo significativo envolvido na reutilização das seringas descartáveis de insulina, as diretrizes da política de saúde deveriam ser transformadas para corresponder com os achados das pesquisas e aparecer na prática corrente ${ }^{(18)}$. Os profissionais de saúde que entendem as razões dos portadores de diabetes reutilizarem as seringas descartáveis deveriam não ser forçados a violar a política governamental na prática. Assim, as políticas institucionais deveriam ser modificadas para permitir que os profissionais informem aos portadores de diabetes, os riscos e benefícios desta prática.

Os profissionais de saúde devem estar informados sobre 
custos do tratamento, necessidades e decisões tomadas pelos portadores de diabetes. Também, devem pesar os riscos e benefícios destas decisões, providenciando orientações baseadas em evidências científicas para assistir os portadores de diabetes na melhor escolha possível quanto a este cuidado ${ }^{(18)}$.

Ao iniciar a prática de reutilização de seringas descartáveis, o portador de diabetes deveria ser avaliado pelo médico, enfermeira, educadores em diabetes. Nesta avaliação, deve-se assegurar a ausência de infecção nos locais de aplicação de insulina e observar que o cliente seja capaz de recolocar o protetor da agulha com segurança. É preciso ainda uma acuidade visual adequada, destreza manual e ausência de tremor. Esta prática é contra-indicada para pessoas com higiene pessoal precária, infecção aguda concorrente, ferida aberta nas mãos, ou diminuição de resistência à infecção ${ }^{(19)}$.

Diante desta complexidade de informações a respeito da utilização e reutilização de seringas descartáveis, as mudanças tecnológicas no que tange aos instrumentais utilizados na administração de insulina, as diferenças culturais, reconhecemos que estudos acerca desta temática devam ser aprofundados e que pesquisas definitivas deveriam ser conduzidas por enfermeiras e outros profissionais a respeito dos riscos e benefícios desta prática.

\section{OBJETIVOS}

1. Caracterizar os portadores de diabetes mellitus segundo variáveis sócio-demográficas, reutilização de seringas e agulhas e a aquisição de seringas descartáveis para insulina;

2. Analisar a freqüência e os custos da prática de reutilização de seringas descartáveis para a administração de insulina pelos portadores de diabetes no domicílio.

\section{MATERIAL E MÉTODOS}

Trata-se de um estudo tipo "survey". Considerando a população base de 1997, quanto ao sexo e faixa etária e os critérios de inclusão, pacientes com idade superior a 18 anos e em uso de insulina, a amostra foi constituída por 113 portadores de diabetes atendidos junto ao Ambulatório de Endocrinologia e Metabologia do Hospital das Clínicas da Faculdade de Medicina de Ribeirão Preto da Universidade de São Paulo (HCFMRP-USP).

$O$ instrumento utilizado para coleta de dados foi um roteiro para entrevista contendo 18 questões semi-abertas versando sobre variáveis sócio - demográficas, reutilização de seringas e agulhas e aquisição de seringas e agulhas descartáveis para insulina. $\mathrm{Na}$ seqüência, o pesquisador dirigiu-se às pessoas portadoras de diabetes esclarecendo a elas o objetivo e a natureza da investigação, obtendo o consentimento formal das mesmas para a coleta de dados e assegurando-lhes 0 anonimato.

A coleta de dados foi realizada no Ambulatório de Endocrinologia e Metabologia do HCFMRP-USP. As entrevistas tiveram duração média de quinze minutos. A coleta de dados foi realizada no período de 01 de junho a 14 de dezembro de 1998. As respostas foram registradas no instrumento pelos entrevistadores, após terem sido submetidos a treinamento prévio, durante a coleta de dados. Para a análise dos dados foi utilizado o Módulo ANALYSIS do EPIINFO versão 6.04b.

\section{RESULTADOS}

Caracterização de portadores de diabetes mellitus atendidos em um hospital de grande porte, segundo variáveis sócio-demográficas

Dos 113 portadores de diabetes entrevistados, $85(75,2 \%)$ são do sexo feminino e 28 (24,8\%) masculino. Quanto a idade, houve uma variação de 18 a 83 anos, com uma média de 52 anos de idade. A maior freqüência encontra-se na faixa etária de 60 a 69 anos $(21,2 \%)$, seguida da faixa de 40 a 49 anos $(20,3 \%)$. Em relação a procedência dos portadores de diabetes $37(32,7 \%)$ são da cidade de Ribeirão Preto, 69 (61,1\%) de outros municípios do Estado de São Paulo e 7 (6,2\%) de outros Estados do país. Em relação a escolaridade e ocupação, 45 (39,8\%) concluiriam a primeira fase do ensino fundamental, 50 (44,2\%) são aposentados e $33(29,2 \%)$ mulheres do lar. A renda familiar mensal variou de 0,5 a 17,6 salários mínimos, sendo que $31(27,4 \%)$ portadores de diabetes referem renda de 1 a 2 salários mínimos. A renda familiar mensal média foi de 3,2 salários mínimos. Quanto ao número de membros na família variou de 1 a 9 , sendo a média de 3 membros por família. Estes dados reforçam a nossa preocupação de estabelecermos estratégias de educação em diabetes considerando as dificuldades financeiras das famílias.

Caracterização de portadores de diabetes mellitus atendidos em um hospital de grande porte segundo variáveis relacionadas à freqüência de reutilização de seringas e agulhas descartáveis de insulina

Em relação ao uso de insulina obtivemos que a maioria dos portadores de diabetes recebe a terapêutica convencional, sendo $36(31,9 \%)$ administram insulina uma vez ao dia e $77(68,1 \%)$ duas vezes ao dia, sendo uma pela manhã e outra a tarde. Constatamos que a maioria $106(94,6 \%)$ dos portadores de diabetes reutilizam a seringa e agulha descartáveis e $6(5,4 \%)$ descartam a seringa e agulha após 0 uso.

Um ponto de análise refere-se à freqüência de reutilização 
das seringas, uma vez que, o número de vezes de uso da mesma seringa poderá determinar as alterações das características físicas, químicas e biológicas originais do produto em decorrência do reprocessamento ${ }^{(2)}$.

Na literatura, as freqüências de reutilização das seringas, são descritas em vezes de uso e também em dias de uso, independente do número de aplicações de insulina. Dessa maneira, aparece a freqüência de reutilização variando de 3 a 60 vezes, e de dias a meses ${ }^{(3-5,13,16,20-21)}$

No que se refere a freqüência de reutilização da seringa $e$ agulha, os dados obtidos apontam que 0 uso da mesma agulha variou de 1 a 20 vezes, e seringa de 1 até 60 vezes. Acreditamos que este fato possa estar relacionado às características das seringas e agulhas que apresentam durabilidade diferenciada durante o processo de reutilização.

As maiores freqüências foram 4 vezes, seguido de 3 vezes, o uso da mesma seringa e agulha, sendo a frequêencia usual referida menor que a encontrada na literatura. Isto talvez, possa ser justificado, pela tendência do mercado em fabricar agulhas mais finas e curtas, proporcionando injeções menos dolorosas, ocorrendo o desgaste precoce do material, quando reutilizado.

Ao indagarmos a frequêencia máxima de reutilização de seringas e agulhas descartáveis, obtivemos a freqüência de 6 vezes. Quando comparamos a freqüência máxima com a usual, podemos perceber que houve um aumento na freqüência de reutilização da seringa e agulha, pois os portadores de diabetes estavam reutilizando a seringa descartável por mais de 7 vezes, o que era previamente estabelecido pelo serviço ${ }^{(22)}$.

A reutilização da seringa descartável pode levar à perda da resistência devido à modificação da matéria prima e perda da nitidez na escala de graduação impressa em seu corpo, podendo ocorrer erro de dosagem da medicação. Outra alteração refere-se ao ajuste entre cilindro, êmbolo e anel de vedação, impedindo o deslizamento adequado do êmbolo ${ }^{(21)}$.

Em relação à agulha, esta perde a afiação das bordas do bisel, a siliconização no corpo da agulha e o sistema de ranhuras na parte interna do canhão. Dessa maneira, pode provocar acidentes com o desprendimento das partes e vazamento do conteúdo ${ }^{(21)}$.

Quanto ao descarte da seringa e agulha que está sendo reutilizada, os dados obtidos mostram que o principal motivo de descarte para $64(60,4 \%)$ portadores de diabetes relaciona-se à agulha. Isto talvez possa estar relacionado à recomendação de alguns profissionais e pela literatura de reutilizar a seringa e agulha até 0 momento em que a agulha torna-se rombuda. Esta orientação pode levar os portadores de diabetes a terem injeções dolorosas, para então, descartarem a agulha.

Em contrapartida, 18 (17\%) descartam a seringa e agulha ao atingir a freqüência usual, independente de dor e/ou a agulha tornando-se rombuda. Em relação à dor, estudo ${ }^{(13)}$ realizado com 37 pacientes que reutilizaram a seringa e a agulha por três vezes consecutivas mostra que 13 referiram dor. Destes, 4 na primeira utilização, 4 na segunda e 5 na terceira, mostrando que a dor pode não estar relacionado ao número de vezes de uso da mesma seringa e agulha, sendo necessário estudos mais conclusivos investigando esta questão.

Por outro lado, investigações conduzidas nesta temática envolvem aspectos legais com os fabricantes de seringas descartáveis, principalmente, porque eles garantem a qualidade do produto comercializado através de testes rigorosos, como também não assumem responsabilidade sobre a reutilização do produto ${ }^{(21)}$.

É importante ressaltar que apenas $4(3,8 \%)$ portadores de diabetes referiram o descarte da seringa baseado na orientação de um profissional da saúde, o que nos leva a pensar que há necessidade de maior participação em programas de educação dos profissionais de saúde e portadores de diabetes em relação a insulinoterapia.

Por estes motivos acreditamos que os portadores de diabetes, principalmente os que reutilizam a seringa descartável, devam ser supervisionados periodicamente nos locais de aplicação de insulina. Esta supervisão deve investigar as complicações locais e as injúrias que esta prática pode ocasionar ao portador de diabetes.

Caracterização de portadores de diabetes mellitus atendidos em um hospital de grande porte segundo variáveis relacionadas à aquisição de seringas descartáveis de insulina

Ao investigar o local de aquisição das seringas utilizadas na administração de insulina, $51(45,1 \%)$ portadores de diabetes referem adquirir na Unidade Básica de Saúde, 42 (37,2\%) na farmácia, 20 (17,7\%) na Unidade Básica de Saúde e farmácia.

Dos 51 portadores de diabetes que adquirem as seringas e agulhas na Unidade Básica de Saúde, $14(27,4 \%)$ consideram a distribuição suficiente, 14 (27,4\%) não e 1 (2\%) não respondeu a questão. Em relação às dificuldades encontradas na aquisição de seringas descartáveis de insulina, $22(43,2 \%)$ referem que a quantidade distribuída depende do profissional de enfermagem responsável pelo fornecimento, sem mencionar critérios estabelecidos, como podemos constatar nas falas abaixo:

Quando as meninas do posto tem, elas dão as seringas e agulhas.(entrev.16)

Depende da moça, tem uma que dá e outra que não. (entrev. 43)

Depende da enfermagem, então eu vou em mais de um posto de saúde para ganhar seringas. (entrev. 97)

Vale ressaltar que o local de estudo, não fornece seringas e insulina aos seus usuários. 
Parece-nos que a falta de normatização quanto ao fornecimento de seringas nas Unidades Básicas de Saúde em nossa Região, e sobretudo no país, fica secundário à problematização da distribuição de medicamentos, dentre eles a insulina. A distribuição de medicamentos sofre a oscilação do fornecimento, da qualidade do produto e a pressão das indústrias farmacêuticas, entre outros.

No entanto, a problemática de distribuição de seringas está presente em nossa realidade. Os dados obtidos mostram que os portadores de diabetes adquirem-nos gratuitamente na Unidade Básica de Saúde em quantidade suficiente para o consumo mensal, mas referem reutilizar as seringas descartáveis para garantir um estoque de seringas e agulhas em casa e enfrentar a oscilação de fornecimento nas instituições de saúde, como podemos ver na seguinte fala:

O que eu ganho no posto é suficiente para o mês, muitas vezes sobra lá em casa. (entrev.13)

De outro modo, encontramos portadores de diabetes que não sabem informar quantas seringas e agulhas adquirem no mês, devido à irregularidade do fornecimento de seringas:

Não é suficiente o que ganho no posto, não dá para o mês. (entrev. 52)

Eu ganhei, mas acaba antes de pegar de novo.(entrev. 34)

Contudo, em ambos os casos, perpassa a reutilização de seringas descartáveis na administração de insulina. Diante desta situação, a Secretaria Municipal de Saúde, em Ribeirão Preto, estabeleceu um protocolo de fornecimento de seringas descartáveis pautado na literatura e prática dos portadores de diabetes na reutilização de seringas ${ }^{(23)}$. Desta maneira, o protocolo estabelece 0 fornecimento de oito seringas/mês para os portadores de diabetes que realizam uma aplicação de insulina diária e o dobro para os portadores que aplicam o medicamento duas vezes ao dia. Outro ponto estabelecido no protocolo refere-se a compra de seringa e agulha conjugada, considerando o comportamento dos portadores de diabetes em lavar as seringas com água para retirar a insulina retida no espaço morto ${ }^{(23)}$. Como o serviço fornece a insulina, este protocolo poderá a longo prazo reduzir custo do fornecimento, pois a cada aplicação de insulina eram desprezadas cinco unidades pelo portador de diabetes.

No entanto, entendemos que ao implementar um protocolo de fornecimento de seringas descartáveis para a administração de insulina, faz-se necessário desenvolver um programa de educação continuada junto aos profissionais de saúde e portadores de diabetes. É preciso sistematizar o processo de compras, garantindo a participação dos setores envolvidos, visando atender os usuários do serviço em relação a custos e adequação dos materiais de consumo nas Instituições de saúde.

Análise dos custos quanto a prática de reutilização de seringas descartáveis para a administração de insulina pelos portadores de diabetes no domicílio

Quanto aos custos, encontramos que aproximadamente metade da população adquire as seringas e agulhas na farmácia com recursos próprios, sendo que o preço referido variou de $R \$ 0,15$ a $R \$ 1,00$, predominando $R \$ 0,50$ o preço de cada seringa e agulha, o que vem ao encontro do preço de mercado.

Analisaremos 0 consumo de seringas e agulhas, considerando o uso único da seringa e agulha descartável. Constatamos que os $36(31,9 \%)$ portadores de diabetes que administram insulina uma vez ao dia, conforme mencionado anteriormente, utilizariam 1.080 seringas ao mês, representando custo diário de $R \$ 18,00$, mensal de $R \$ 540,00$ e $R \$ 15,00$ por portador de diabetes. Quanto aos $77(68,1 \%)$ que administram insulina duas vezes ao dia, consumiriam 154 seringas ao dia, totalizando 4.620 seringas no mês, com custo diário de $R \$ 77,00$, mensal de $R \$ 2.310,00$ e $R$ \$ 30,00 por portador de diabetes.

Desse modo, ao utilizar uma seringa descartável para cada aplicação de insulina, o consumo diário seria de 190 seringas descartáveis por essa população, perfazendo um total de 5.700 seringas descartáveis no mês, representando um custo de $R \$ 95,00$ diário e mensal de $R \$ 2.850,00^{*}$, e a média de $R \$ 25,22 /$ mês por pessoa

Em relação ao consumo de insulina, encontramos um total de 5.306 U.I. de insulina aplicada por dia, e 159.180 U.I. de insulina no mês, o que equivale a 159,18 frascos de insulina, representando um custo mensal de $R \$ 2.387,70^{* *}$ e a média de $R \$ 21,13 /$ mês por pessoa.

Portanto, ao considerarmos o custo do consumo mensal envolvendo a aquisição de seringas descartáveis e insulina, evidenciamos o equivalente a $R \$ 2.850,00$ em seringas mais $R \$$ $2.387,70$ em insulina, perfazendo um total de $R \$ 5.237,70$, sendo 0 gasto médio por portador de diabetes mellitus de $\mathrm{R} \$ 46,35$. Frente ao salário mínimo*** encontramos que 0 gasto com seringas descartáveis e insulina representa $35,65 \%$ do referido salário; portanto, o mínimo que um portador de diabetes gasta para o seu tratamento fica em torno de $36 \%$ do salário - mínimo vigente no país. Mas, quando acrescenta ao tratamento destes pacientes os itens insulina, seringas descartáveis, e fitas reagentes, alcança-se até 70\%

\footnotetext{
* preço unitário da seringa descartável no mercado $=\mathrm{R} \$ 0,50(12 / 98)$

** preço unitário médio do frasco de insulina no mercado $=\mathrm{R} \$ 15,00(12 / 98)$

*** Baseando-se no salário mínimo de R\$130,00, vigente em maio de 1998 (Gazeta Mercantil, 10 mai. 1998)
} 
do salário mínimo em gastos para o seu controle metabólico ${ }^{(24)}$. Estes custos nos preocupam, uma vez que, a insulinoterapia é uma das facetas do tratamento em diabetes, e representa os custos econômicos diretos no tratamento, ou seja, recursos gastos na prevenção, diagnóstico e tratamento.

Os custos econômicos diretos no tratamento em diabetes incluem os gastos com transportes, consultas médica, enfermagem, nutricionista, exames complementares, medicamentos, dietas, fitas reagentes para glicemia, cetonúria entre outras despesas mensais que podem superar o orçamento familiar ${ }^{(6)}$. Os custos indiretos seriam aqueles que impossibilitariam os portadores de diabetes de participarem na produção de bens e serviços ${ }^{(24)}$.

Recomenda-se a participação dos profissionais de saúde investindo na educação para o auto - cuidado do portador de diabetes em seu domicilio, objetivando diminuir os custos indiretos que as complicações crônicas impõem aos serviços de saúde e sociedade, aí envolvida a reutilização de seringas descartáveis ${ }^{(24)}$. Desse modo, analisaremos o custo do consumo mensal de seringas por portador de diabetes, considerando a prática de reutilização de seringa descartável encontrada nesta investigação, estabelecendo a freqüência de quatro vezes a utilização da mesma seringa.

Evidenciamos que 36 portadores de diabetes que administram insulina uma vez ao dia, reutilizando uma seringa $e$ agulha por quatro vezes, conforme a freqüência de uso relatada neste estudo, consumiriam individualmente oito seringas ao mês. Assim, esses portadores de diabetes consumiriam um total de 288 seringas no mês, representando um custo total mensal de $R \$ 144,00$ e $R \$$ 4,00 pessoa/mês.

Em relação aos 77 portadores de diabetes que administram insulina duas vezes ao dia, reutilizando uma seringa e agulha por quatro vezes, haveria um consumo mensal de 15 seringas por portador de diabetes. Desse modo, consumiriam um total de 1.155 seringas no mês, a um custo de $R \$ 577,50 /$ mês e $R \$ 7,50$ por pessoa/ mês.

Assim, os 113 portadores de diabetes consumiriam 1.443 seringas no mês, representando um custo de $R \$ 721,50$ /mês, e a média de $\mathrm{R} \$ 6,38$ por portador de diabetes/mês. Em contrapartida, se utilizassem uma seringa e agulha por aplicação, conforme mencionado anteriormente, o custo mensal seria de $\mathrm{R} \$ 2.850,00$. Podemos observar que com a adoção da prática de reutilização de seringas e agulhas por 4 vezes, conforme o uso referido por esta população, haveria uma diminuição no custo do tratamento dessa população de $R \$ 2.128,50$, representando $74,68 \%$ de redução nos custos com seringas e agulhas em insulinoterapia.

Desta maneira, manifesta-se a redução do custo no tratamento em diabetes, relacionado a reutilização de seringas descartáveis. Assim, parece que esta prática realizada pelos portadores de diabetes pode estar fundamentada em causas econômicas, principalmente, considerando os dados sócio demográficos obtidos.

Assim, a distribuição dos medicamentos parece não solucionar os problemas de saúde pública no Brasil, principalmente, pelas grandes variações regionais, assim como sabemos que a saúde pública está atrelada a melhores condições sócio - econômicas da população, melhor alimentação, moradias com água tratada e esgoto sanitário, e medidas de ordem profilática como educação, cuidados de higiene e vacinações ${ }^{(25)}$.

Consideramos, portanto, que a problemática de reutilização de seringas descartáveis parece não ser resolvida somente com a distribuição gratuita de seringas descartáveis. Faz-se necessário um aprofundamento na avaliação desta prática, com estudos conclusivos que possam fundamentar a orientação a ser realizada pelos profissionais de saúde.

Não há perigo significativo na reutilização de seringas descartáveis na aplicação de insulina; isto deveria aparecer na prática de saúde e as diretrizes políticas deveriam ser modificadas para corresponder aos achados de pesquisa ${ }^{(18)}$. Portanto, o educador em diabetes juntamente com o portador de diabetes ao realizar a opção pelo instrumental, dentre eles, a seringa descartável, deve considerar a possibilidade de sua reutilização, e alertar quanto aos riscos e benefícios desta prática. No entanto, esta diretriz deveria estar normatizada pelo Ministério da Saúde, evitando que os profissionais violem a portaria da DIMED 04/86 vigente, surgindo urgente a necessidade de sua revisão.

Assim, acreditamos que a educação em diabetes voltada para a reutilização de seringas descartáveis durante a aplicação de insulina é uma das facetas na assistência a saúde do portador de diabetes, e que merece a atenção fundamentada em todos os aspectos que foram discutidos no presente estudo.

\section{CONSIDERAÇÕES FINAIS}

A divulgação dos resultados do Diabetes Control and Complication Trial Research Group - DCCT representou um marco para repensar a educação em diabetes em nível mundial. Em relação à terapia insulínica, em especial, constata-se um grande movimento na tentativa de substituir a terapêutica convencional pela intensiva, constituindo um desafio para os profissionais de saúde e portadores de diabetes mellitus, exigindo esforços conjuntos para que atinjam um bom controle metabólico a fim de evitar ou minimizar as complicações crônicas a longo prazo.

Estes esforços devem ser direcionados para auxiliar os portadores de diabetes mellitus a incorporar a terapêutica intensiva de insulina, em particular, aos aspectos relacionados ao instrumental 
utilizado na administração de insulina e reforçar a sua capacitação para a auto - aplicação da insulina.

Neste sentido, o conjunto de dados obtidos nesta investigação mostrou-nos que a maioria dos portadores de diabetes mellitus tem utilizado para a seringa descartável e que a prática de reutilização de seringas descartáveis está incorporada no seu cotidiano.

Esta investigação mostrou que os portadores de diabetes, em sua maioria, recolocam o protetor da agulha após o uso, conforme a orientação da Associação Americana de Diabetes - ADA, relatando a freqüência de 4 seguido de 3 vezes o uso da mesma seringa.

Os dados encontrados nesta investigação merecem ser analisados pelos profissionais de saúde visando 0 aprimoramento dos programas de educação e assistência à pessoas portadoras de diabetes. Neste sentido, delimitamos algumas recomendações a seguir: revisão da recomendação do DIMED - Portaria n. ${ }^{\circ} 4$ de 07 de fevereiro de 1986, baseada em evidências científicas e da recomendação da Associação Americana de Diabetes; formação e

\section{REFERÊNCIAS BIBLIOGRÁFICAS}

1. Ministério da Saúde (BR). Portaria $n^{0} 03$ de 07 de fevereiro de 1986. Diário Oficial da União 1986 a 12 fev:Seção 1; 2.326.

2. Ministério da Saúde (BR). Portaria $n^{0} 04$ de 07 de fevereiro de 1986. Diário Oficial da União 1986b 12 fev: Seção 1, 2.327.

3. Collins BJ, Spence BK, Richardson SG, Hunter J. Safety of reusing disposable plastic insulin syringes. Lancet 1983; 1:559-60.

4. Bloom A. Syringes for diabetics. Br Med J 1985 Mar; 290:727-8. 5. McCarthy JA, Sink PF, Covarrubias BM. Reevalution of single-use insulin syringes. Diabetes Care 1988; 11(10):817-8.

6. Hissa MN, Montenegro RM, Colares FAN. Reutilização de seringas descartáveis de insulina: diminuição de custos no tratamento do diabetes insulino-dependente. Arq Bras Endocrinol Metabol 1987 dez.; 31(4):78-80.

7. Jackson PG, Keen H, Noble CJ, Simmons NA. Injection abscesses in a diabetic due to Mycobacterium chelonei var abscessus. Br Med J 1980; 281:1105-6.

8. Black HJ, Hart FD. Plastic insulin syringes. Br Med J 1988 Apr.; 296:1195.

9. Toal FM. Disposable needles for diabetics. Lancet 1978 Jan.; 7:445.

10. Greenough A, Cockcroft PM, Bloom A. Disposable syringes for insulin injection. Br Med J 1979; 1(6176):1467-8.

11. Swift PGF, Hearnshawn JR. Insulin injections and infections. $\mathrm{Br}$ Med J 1981; 282:340. capacitação de equipes multiprofissionais que atendam as necessidades e possibilidades reais dos portadores de diabetes quanto à aquisição do instrumental para auto - aplicação de insulina; desenvolvimento de pesquisas no sentido de identificar os fatores que possam beneficiar e/ou causar riscos ou injúrias aos portadores de diabetes mellitus na reutilização de seringas descartáveis de insulina; estabelecimento de protocolos de freqüência de reutilização de seringas, procedimento de reutilização na administração de insulina, fundamentado na literatura; estabelecimento de diretrizes para distribuição de seringas descartáveis considerando os fatores econômicos, antropológicos e riscos biológicos; reconhecimento do enfermeiro, responsável pelo acompanhamento do portador de diabetes mellitus, comprometendo-se com a educação e treinamento em relação ao instrumental para auto - aplicação de insulina; fortalecimento e ampliação de grupos de educação em diabetes, os quais motivam os portadores de diabetes mellitus à participação efetiva, criando oportunidade de elaboração dos problemas enfrentados.

12. Dankert J, Drayer NM. Sterility problems and the diabetic. Lancet 1978; 10:1256.

13. Bosquet F, Grimaldi A, Thervet F. Insulin syringe reuse. Diabetes Care 1986; 9(3):310.

14. Scain SF. Reutilização de seringa descartável para aplicação de insulina. Rev HCPA 1985 dez; 5(2):181-8.

15. Rezende CH, Lima MG. Avaliação do uso de seringas descartáveis em diabéticos no Hospital do Servidor Público Municipal de São Paulo. São Paulo (SP); 1991.

16. Strathclyde Diabetic Group. Disposable or non-disposable syringes and needles for diabetics?. Br Med J 1983; 286:369-70.

17. Alexander WD, Corrigan C, Todd P, Wells M. Disposable of plastic insulin syringes and needles. Br Med J 1987 Aug.; 295:527.

18. Poteet WG, Reinert B, Ptak H. Outcome of multiple usage of disposable syringes in the insulin-requiring diabetic. Nurs Res 1987 Nov-Dec.; 36(6):350-3.

19. American Diabetes Association. Insulin administration (Position Statement) Diabetes Care ?serial online? 1999 [Cited 1999 fev 04]; 22(Suppl. 1): (S83 screens). Alexandria. Avaliable from: URL: http:// www.diabetes.org.br.

20. Lester E, Woodroffe FJ, Grant AJ. Experience with routine reuse of plastic insulin syringes. Br Med J 1984; 289:1498-9.

21. Daschner F. Reutilização de Artigos hospitalares. In: Rodrigues ACR, Mendonça JS, Amarante JMB, Alves Filho MB, Grinbaum RS, et al. organizadores. Infecções hospitalares: prevenção e controle. São Paulo (SP):Sarvier; 1997. p.440-6. 
22. Damasceno MMC, Rocha TJ, Lino RLP, Carvalho ZMF Problemas identificados na clientela diabética: análise quantitativa. Rev Enfermagem UERJ 1995 maio; 3(1):19-28.

23. Secretaria Municipal da Saúde (RP). Protocolo de fornecimento e reutilização de seringa descartável na insulinoterapia de pacientes diabéticos. Ribeirão Preto (SP): Secretaria Municipal da Saúde; 1998.
24. Scain SF, Oliveira CH, Franzen E. Aspectos de assistência de Enfermagem ambulatorial no custo do tratamento de diabéticos. Rev Gaúch Enfermagem 1987 jul.; 8(2):149-67.

25. Saraiva JL. Política nacional de medicamentos. Rev Bras Educ Méd 1983; 7(3):163-78. 